\title{
Emission from the Ionized Gaseous Halos of Low-redshift Galaxies and Their Neighbors
}

\author{
Huanian Zhang (iD), Dennis Zaritsky (iD), and Peter Behroozi (iD \\ Steward Observatory, University of Arizona, Tucson, AZ 85719, USA; fantasyzhn@email.arizona.edu \\ Received 2018 February 12; revised 2018 May 10; accepted 2018 May 16; published 2018 June 28
}

\begin{abstract}
Using a sample of nearly half a million galaxies, intersected by over 8 million lines of sight from the Sloan Digital Sky Survey Data Release 12, we extend our previous study of the recombination radiation emitted by the gaseous halos of nearby galaxies. We identify an inflection in the radial profile of the $\mathrm{H} \alpha+\mathrm{N}$ [II] radial emission profile at a projected radius of $\sim 50 \mathrm{kpc}$ and suggest that beyond this radius the emission from ionized gas in spatially correlated halos dominates the profile. We confirm that this is a viable hypothesis using results from a highly simplified theoretical treatment in which the dark matter halo distribution from cosmological simulations is straightforwardly populated with gas. Whether we fit the fraction of halo gas in a cooler $(T=12,000 \mathrm{~K})$, smooth $(c=1)$ component $\left(0.26\right.$ for galaxies with $M_{*}=10^{10.88} M_{\odot}$ and 0.34 for those with $\left.M_{*}=10^{10.18} M_{\odot}\right)$ or take independent values of this fraction from published hydrodynamical simulations ( 0.19 and 0.38 , respectively), this model successfully reproduces the radial location and amplitude of the observed inflection. We also observe that the physical nature of the gaseous halo connects to primary galaxy morphology beyond any relationship to the galaxy's stellar mass and star formation rate. We explore whether the model reproduces behavior related to the central galaxy's stellar mass, star formation rate, and morphology. We find that it is unsuccessful in reproducing the observations at this level of detail and discuss various shortcomings of our simple model that may be responsible.
\end{abstract}

Key words: galaxies: halos - intergalactic medium - large-scale structure of universe

\section{Introduction}

Tracing the circumgalactic medium (CGM) is a longstanding goal with correspondingly extensive literature (see Tumlinson et al. 2017 for a recent comprehensive review). The importance of the CGM arises from the expectation that it is the reservoir for subsequent star formation in galaxies (Spitzer 1956), the depository for outflowing material, and the site of the majority of the baryons in galaxies (Bregman 2007; Werk et al. 2014).

We have recently turned a corner in our observational study of the CGM by advancing from studies that produced ever decreasing upper limits on the resulting recombination emission line flux from this gas (Bland-Hawthorn et al. 1997; Dicaire et al. 2008; Christlein et al. 2010; Adams et al. 2011; Hlavacek-Larrondo et al. 2011) to detections of this emission utilizing either new techniques (Zhang et al. 2016, hereafter Paper I) or new instrumentation (Bellhouse et al. 2017; Fumagalli et al. 2017).

Of specific relevance to this study is our previous detection of $\mathrm{H} \alpha+\mathrm{N}[\mathrm{II}]$ emission surrounding low-redshift galaxies out to projected radii of $100 \mathrm{kpc}$, described in Paper I, using a sample of over 7 million lines of sight from SDSS DR12 (Alam et al. 2015). Using the same data source and technique, we now turn to questions beyond detection and basic characterization. Specifically, we explore the shape of the stacked emission line radial profile and relationships between galaxy properties such as stellar mass, star formation rate (SFR), morphology, and environment and this radial profile. Our aim is to uncover variations among halo properties that can be used to identify the dominant ionization source and to constrain the cold gas distribution, both of which will lead to a greater understanding of the physical conditions of the gas.

We identify an apparent inflection in the radial profile of the recombination line emission from halo gas (Section 2). We confirm that this inflection is not due to uncertainties in the background measurement (Section 2), and using highly simplified models of the halo gas embedded within a distribution of dark matter halos produced from cosmological simulations, we demonstrate that the radial location and amplitude of the inflection are consistent with expectations arising from the consideration of the flux contributed by spatially correlated halos (Section 3).

We explore the models further by examining correlations between the radial emission line profiles and various central galaxy properties (Section 2) and expectations drawn from the models (Section 3). Here, we are less successful at reproducing the observations, but this disagreement likely reflects deficits in our simple models. The comparison to our models is nevertheless helpful to guide intuition, but more physically realistic simulations are absolutely critical for achieving a robust and detailed understanding of the findings described here. We adopt standard cosmological parameters $\Omega_{m}=0.3, \Omega_{\Lambda}=0.7$, $\Omega_{k}=0$, and the dimensionless Hubble constant $h=0.7$.

\section{Data Analysis and Results}

We follow the approach developed in Paper I. We obtain spectra from the Sloan Digital Sky Survey Data Releases (Alam et al. 2015, SDSS DR12), but now we decrease the lower bound on the primary galaxy redshift range from 0.05 to 0.025 to increase the available number of lines of sight by roughly 1 million. Briefly, as described in Paper I, we classify galaxies that meet the criteria in redshift (now from $0.025<z<0.2)$, luminosity $\left(10^{10}<L / L_{\odot}<10^{11}\right)$, and size $\left(2<R_{50} / \mathrm{kpc}<10\right)$ as primary galaxies, and then study lines of sight to other galaxies that are within $1.5 \mathrm{Mpc}$ projected separation of a primary galaxy and that were SDSS spectroscopic targets. For each such spectrum, we fit and subtract a 10th order polynomial to a $300 \mathrm{~A}$ wide section surrounding the 
Table 1

Measured Flux within Each Radial Bin

\begin{tabular}{lc}
\hline \hline $\begin{array}{l}r_{p} \\
{[\mathrm{kpc}]}\end{array}$ & $f$ \\
\hline 5 & $(2.75 \pm 1.65) \times 10^{-2}$ \\
10 & $(2.56 \pm 0.40) \times 10^{-2}$ \\
18 & $(5.10 \pm 1.51) \times 10^{-3}$ \\
32 & $(1.39 \pm 0.71) \times 10^{-3}$ \\
58 & $(1.04 \pm 0.39) \times 10^{-3}$ \\
107 & $(7.03 \pm 2.15) \times 10^{-4}$ \\
195 & $(3.26 \pm 1.16) \times 10^{-4}$ \\
343 & $(2.08 \pm 0.68) \times 10^{-4}$ \\
602 & $(1.55 \pm 0.38) \times 10^{-4}$ \\
936 & $(1.29 \pm 0.30) \times 10^{-4}$ \\
1310 & $(1.07 \pm 0.24) \times 10^{-4}$ \\
\hline
\end{tabular}

wavelength of $\mathrm{H} \alpha$ at the primary galaxy redshift to remove the continuum. We then measure the residual $\mathrm{H} \alpha+\mathrm{N}[\mathrm{II}]$ flux within a velocity window corresponding to $\pm 275 \mathrm{~km} \mathrm{~s}^{-1}$ from the primary galaxy to capture the majority of the emission flux from the halo gas. To avoid having contamination by satellites of the target galaxy, we require the redshift difference between the primary and the line-of-sight target to be greater than 0.05 and we require that $|f| \leqslant 0.3 \times 10^{-17} \mathrm{erg} \mathrm{cm}^{-2} \mathrm{~s}^{-1} \AA^{-1}$, as in our previous work. The closeness in wavelength of $\mathrm{H} \alpha$ and $\mathrm{N}$ [II], our inability to identify the emission lines in individual spectra, and the unknown peculiar velocity of the halo gas relative to the primary galaxy, preclude us from separating the flux from these lines in our measurement. We apply one final correction for a possible remaining systematic residual in the subtracted sky spectra by combining all of the spectra in the observed frame, fitting a 10th order polynomial, and subtracting the integral of that fit over the measurement window.

Combining all of our final measurements from over 8 million lines of sight, sorted in radial bins, we obtain the radial profile of the $\mathrm{H} \alpha+\mathrm{N}[\mathrm{II}]$ emission flux, $f$, around the average primary galaxy. ${ }^{1}$ The data plotted in Figure 1 represent the mean values within each radial bin and the quoted uncertainty is the statistical uncertainty in that mean value derived from the dispersion of measurements in each bin. We present the plotted values in Table 1.

A critical aspect of this analysis, in which we are attempting to extend our study to the largest possible projected radius, is the determination of the far-field value of $f$, or the "background" level, $f_{B}$. In Paper I, we measured $f_{B}$ using the average of $f$ at large radii $\left(1<r_{p} / \mathrm{Mpc}<1.5\right)$ and then presented and discussed the net emission flux, $\Delta f \equiv f-f_{B}$, as a function of projected radius, $r_{p}$. Here, because we are interested in the shape of the outermost emission line flux radial profile and the possible flux contribution by the nearby environment, we are concerned that subtracting a single, uncertain $f_{B}$ value for all systems will inadvertently mask behavior of interest underneath a large uncertainty in the net emission flux.

To estimate the value of $f_{B}$ and its associated uncertainty, we first follow our approach from Paper I and measure $f_{B}$ from lines of sight at $1.0<r_{p} / \mathrm{Mpc}<1.5$, where we expect the emission to have dropped below our detection threshold. We measure $f_{B}=1.09 \times 10^{-21} \mathrm{erg} \mathrm{cm}^{-2} \mathrm{~s}^{-1} \AA^{-1}$. This non-zero result implies that we are either incorrect about our assumption that

\footnotetext{
1 The conversion factor to units between the values we present and those used commonly in the literature, $\operatorname{erg~cm}^{-2} \mathrm{~s}^{-1} \operatorname{arcsec}^{-2}$, is 1.7 .
}

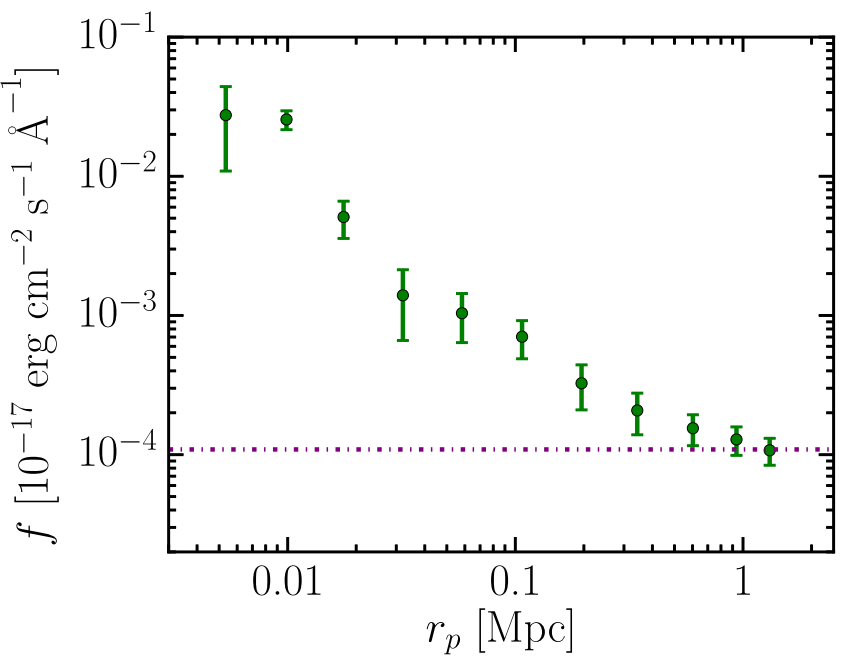

Figure 1. Radial profile of $\mathrm{H} \alpha+\mathrm{N}[\mathrm{II}]$ flux, $f$, for the entire data sample and the indication of the systematic limit on our detection based on the value obtained at large separation, $f_{B}$ (horizontal dotted line). Flux is significantly detected to beyond $100 \mathrm{kpc}$, but the character of the profile appears to change from steeply declining to moderately declining at $\sim 50 \mathrm{kpc}$. We explore the significance of this claim and present our interpretation of this change of behavior in Section 3.

Table 2

Measured Flux within Each Radial Bin for Low-mass and High-mass Subsamples

\begin{tabular}{lcc}
\hline \hline $\begin{array}{l}r_{p} \\
{[\mathrm{kpc}]}\end{array}$ & $f\left(M_{*}>10^{10.45} M_{\odot}\right)$ \\
{$\left[10^{-17} \mathrm{erg} \mathrm{cm}^{-2} \mathrm{~s}^{-1} \AA^{-1}\right]$}
\end{tabular}

there is no detectable $\mathrm{H} \alpha+\mathrm{N}[\mathrm{II}]$ flux at such large separation or that there is a systematic uncertainty in our sky and continuum subtraction of comparable magnitude. To distinguish between these possibilities, we extend our examination by shifting the measurement window $\sim 100 \AA$ either blueward or redward, where there are no emission lines, and measure $f_{B}$ again from the lines of sight at large separation. For the blueward window shift we measure $f_{B}=1.01 \times 10^{-21} \mathrm{erg} \mathrm{cm}^{-2} \mathrm{~s}^{-1} \AA^{-1}$ and for the redward shift we measure $f_{B}=-0.85 \times 10^{-21} \mathrm{erg} \mathrm{cm}^{-2} \mathrm{~s}^{-1} \AA^{-1}$. The discrepant results indicate that there is systematic error in $f_{B}$ at the level of $\sim \pm 1 \times 10^{-21} \mathrm{erg} \mathrm{cm}^{-2} \mathrm{~s}^{-1} \AA^{-1}$, so our initial measurement of $f_{B}$ provides no evidence of emission flux at $r_{p}>1 \mathrm{Mpc}$. We conclude that the measured $f_{B}$ represents a systematics-limited detection floor for our stacked lines of sight, thus we do not subtract it.

In Figure 1 we present the radial emission line flux profile from our stacked and radially binned measurements of $f$. In addition, we highlight the detection floor attributable to systematic uncertainties in $f_{B}$. We summarize the findings so far as (1) there is detectable emission, well above the $f_{B}$ limit, out beyond $100 \mathrm{kpc}$, (2) there is a steep decline in the profile from the central galaxy to $\sim 50 \mathrm{kpc}$, and (3) there is an intermediate region from $\sim 50 \mathrm{kpc}$ extending beyond $100 \mathrm{kpc}$ where the profile flattens but remains above $f_{B}$. The inflection between the steep inner decline and the flatter outer region is what we consider to be evidence for emission from spatially correlated halos and what we discuss in more detail in Section 3. If the reader is somewhat skeptical of our claim that the profile changes character at $r_{p} \sim 50 \mathrm{kpc}$, we request 
their indulgence until we present the calculated emission profile from cold gas in an isolated halo.

\subsection{The Emission Line Profile and Primary Galaxy Properties}

To explore the dependencies of the $\mathrm{H} \alpha+\mathrm{N}[\mathrm{II}]$ emission flux on galaxy properties further than we did in Paper I, we extract measures of the galaxy's Sérsic index $(n)$ and absolute magnitude $(M)$ from Simard et al. (2011), stellar mass $\left(M_{*}\right)$ from Kauffmann et al. (2003a, 2003b) and Gallazzi et al. (2005), and current SFR from Brinchmann et al. (2004).

\subsubsection{Dependence on Stellar Mass}

We begin our exploration by considering variations with stellar mass. A natural expectation is that more massive galaxies have more halo gas and therefore a brighter and more extended emitting halo. To test this expectation, we divide the primary galaxies into two nearly equally sized subsamples by setting the demarcation at a stellar mass of $10^{10.45} M_{\odot}$. To quantify any differences between the two samples we compare the mean values of $f$ in units of $10^{-17} \mathrm{erg} \mathrm{cm}^{-2} \mathrm{~s}^{-1} \AA^{-1}$ at radii of 10,50 , and $300 \mathrm{kpc}$ (Table 2).

The mean $f$ values show some statistically significant differences between mass samples, but only at the smallest radii and not perhaps in the expected sense. It is the low stellar mass galaxies that show the larger emission fluxes in the $10 \mathrm{kpc}$ bin. We interpret this result to mean that late-type, star-forming galaxies, which are generally of lower stellar mass, have more luminous halos at these radii. By $50 \mathrm{kpc}$ the fluxes are nearly indistinguishable, suggesting whatever connection existed between the emission and primary galaxy is absent at these, and larger, radii. We draw two conclusions from this initial comparison. First, significant and interesting differences in halo emission line properties are detectable among galaxy subsamples. Second, although we selected by stellar mass, the differences are likely tied to a variety of factors that have different levels of influence as a function of $r_{p}$ and may be difficult to unravel if one cannot control for these factors.

\subsubsection{Dependence on SFR}

One of our goals is to identify the source(s) of ionizing photons for this emitting gas. Escaping high-energy photons from the primary galaxy are one possibility. If so, the strength of the measured emission flux could correlate with the SFR. Furthermore, such a scenario could explain our previous result in which the more massive galaxies, which are likely to be dominated by earlier types, have less emission flux at small $r_{p}$ than do the lower-mass galaxies, which are likely to be dominated by later types. Quantifying such a correlation could lead to a measurement of the escape fraction, which would in and of itself be a significant and valuable result.

To search for a connection between the emission line profile and the SFR of the primary galaxy, we divide the sample nearly equally into a low SFR subsample (SFR $<0.70 M_{\odot} \mathrm{yr}^{-1}$ ) and a high SFR one (SFR $\left.>0.70 M_{\odot} \mathrm{yr}^{-1}\right)$. The demarcation is chosen only to balance the size of the subsamples, not for any physically motivated reason.

We now know that stellar mass might also be a physical driver of differences in the flux profiles, so we control for stellar mass differences. We construct stellar-mass-matched subsamples sorted by SFR by first binning the low and high SFR subsamples by stellar mass. For each stellar mass bin, we include all of the primary galaxies in whichever SFR subsample has fewer galaxies and randomly select an equally sized sample from the other set. We do this for all of the mass bins to obtain one realization of mass-matched low and high SFR subsamples and measure the average of $f$ in bins centered at $r_{p}=10,50$, and $300 \mathrm{kpc}$. The whole process is repeated 2000 times to obtain the distributions of $f$ at the three different radii presented in Figure 2.

The results highlight three qualitatively different radial regimes. At small $r_{p}, 10 \mathrm{kpc}$, the emission in the high SFR subsample is a few times higher than that in the low SFR subsample. We interpret this as the result of the higher SFR and suggest that it arises from a combination of a stronger ionizing radiation field generated by the star formation and a more massive cold gas reservoir. Although these are plausible explanations, physical reality is likely to be complicated by the detailed internal geometry of the central galaxy. Studies of the Ly $\alpha$ emission from starbursting systems have highlighted the complex role of dust content and geometry on the escaping radiation (Hayes et al. 2013, 2014; Bridge et al. 2017). At intermediate $r_{p}, 50 \mathrm{kpc}$, the emission flux is becoming more similar in the two subsamples and therefore less dependent on the SFR of the central galaxy. We interpret this result as indicating that any escaping radiation has a declining effect, for normal low- $z$ galaxies, on the ionization state of the halo gas beyond several tens of kiloparsecs. A corollary to this inference is that the ionization at this and larger radii is, to a greater degree, due to other sources of ionization such as the intergalactic radiation field or to shock heating. Finally, and perhaps somewhat puzzling at first is the reversal seen at the largest $r_{p}, 300 \mathrm{kpc}$, where the emission flux is greater in the subsample of primaries with lower SFR.

We conclude that in mass-controlled subsets, we find that the emission line fluxes of galaxies at $r_{p} \sim 10 \mathrm{kpc}$ are strongly related to the SFR of the primary galaxy, but that beyond $\sim 50 \mathrm{kpc}$ any effects of the primary galaxy are weaker and that differences, where they exist, are likely due primarily to external agents.

Next, we control for differences in morphology, in addition to controlling for stellar mass. As a proxy for morphology we use the Sérsic index (Sérsic 1968), classifying those primaries with $n>3$ as spheroids and $n \leqslant 2$ as disks. We present the $f$ distributions at the three selected radii in Figure 3.

The pattern of results is somewhat similar to the earlier one, with the more strongly star-forming galaxies having larger fluxes at 10 and $50 \mathrm{kpc}$, although at $300 \mathrm{kpc}$ the ordering changes relative to the previous results. We conclude that 1) the local properties that drive the emission (local ionization field, halo gas density distribution, gas temperature) are different both in the halo of the primary galaxy and in the local environment for high and low star-forming galaxies and 2) primary galaxy morphology contains additional information about the physical nature of the CGM beyond that arising from its relationship to the SFR.

\section{Theoretical Models and Discussion}

We base our modeling on halo merger trees from the Bolshoi-Planck simulation (Klypin et al. 2016; RodríguezPuebla et al. 2016), a dark-matter-only simulation with a comoving side length of $250 \mathrm{Mpc} h^{-1}$, mass resolution of $1.8 \times 10^{8} M_{\odot}\left(2048^{3}\right.$ particles $)$, and force resolution of $1 \mathrm{kpc} h^{-1}$. Halos were found with the ROCKSTAR phase-space 


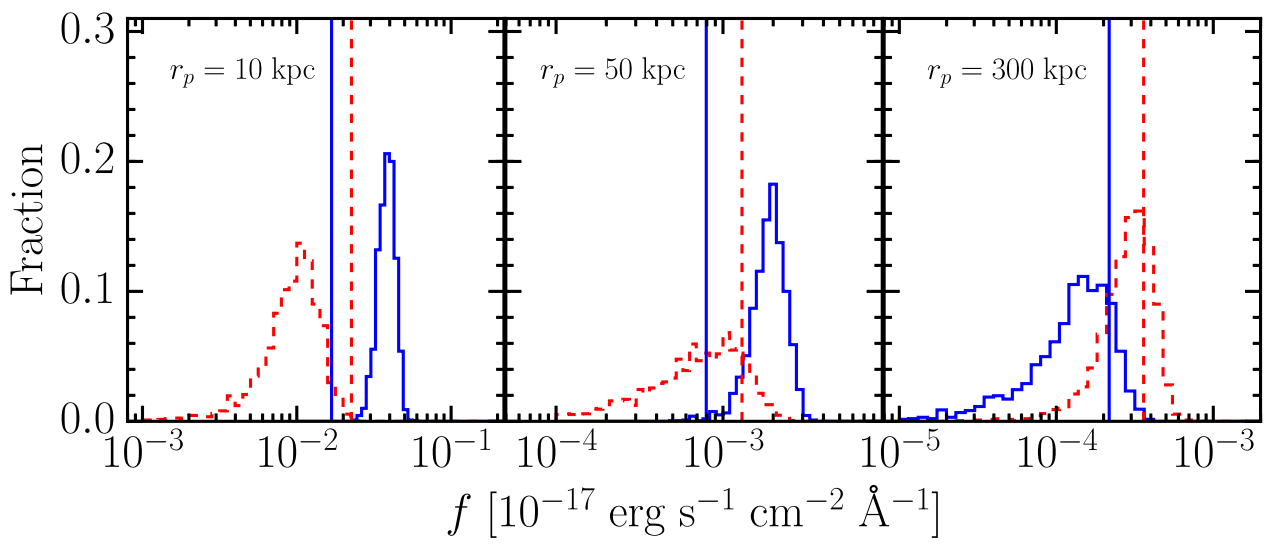

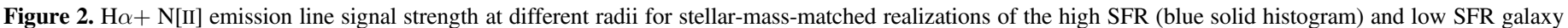

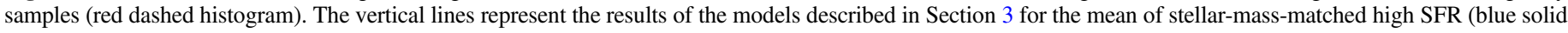
line) and low SFR samples (red dashed line).

halo finder (Behroozi et al. 2013), and merger trees were generated with the CONSISTENT TREES code (Behroozi et al. 2013). The simulation adopted a flat, $\Lambda$ CDM cosmology with $\Omega_{M}=0.307, \Omega_{B}=0.048, h=0.678, n_{s}=0.96$, and $\sigma_{8}=0.823$, similar to recent Planck constraints (Planck Collaboration et al. 2013).

Galaxy stellar masses and SFRs were modeled with the UNIVERSEMACHINE code (P. Behroozi et al. 2018, in preparation). Briefly, the code empirically determines how galaxy SFRs should depend on host dark matter halo mass, halo accretion rate, and redshift so that many observables are reproduced. For this project, we used catalogs at $z=0.1$ (the median of the observed target galaxies) that match observed galaxy stellar mass functions, specific SFRs, quiescent fractions, and correlation functions (split by color, mass, and redshift). Because the code does not currently output galaxy $r$ band luminosities or sizes, we matched mock galaxies to the closest SDSS galaxies in stellar mass and SFR. The mock catalogs thus reproduce by definition the joint probability distribution for $r$-band luminosity and galaxy size, given galaxy stellar mass and SFR.

Once we have a catalog of halos and the galaxies associated with them, we populate each halo with gas. Within each NFW dark matter halo (Navarro et al. 1997) we adopt a hydroequilibrium isothermal model as the description of the gas mass density profile:

$$
\rho_{g}(r)=h_{f} \rho_{0} \exp \left[-\Delta_{\mathrm{NFW}}\left(1-\frac{\ln \left(1+r / r_{s}\right)}{r / r_{s}}\right)\right],
$$

where $\Delta_{\mathrm{NFW}}=4 \pi \mathrm{G} \rho_{0} r_{s}^{2} \mu m_{p} /\left(k_{\mathrm{B}} T_{\mathrm{vir}}\right), h_{f}$ is the hydrogen gas mass fraction, $\mu$ is mean molecular weight, $m_{p}$ is the proton mass and $T_{\text {vir }}$ is the virial temperature (Capelo et al. 2010). To estimate $h_{f}$, we adopt the cosmological baryon fraction, $\simeq 0.16$ (Planck Collaboration et al. 2013), multiply it by the fraction of baryons in a galaxy that are in the halo as opposed to being in the stars and interstellar medium of the central galaxy $(\sim 0.85$; Behroozi et al. 2010; McGaugh et al. 2010; Tumlinson et al. 2017) and then by the fraction of that baryonic mass that is in hydrogen atoms, $\sim 0.75$. The dominant uncertainty in this calculation comes from the adopted fraction of the baryons that are in the halo, which could be as low as 0.6 but probably not much higher than 0.85 (see Zaritsky \& Courtois 2017, and references therein for a recent baryon accounting in the Milky
Way). For primordial gas, $\mu$ is $\sim 0.59$ because metals are trace elements and the hydrogen/helium mass ratio is $3: 1$. Even though the halo is metal-enriched (Tumlinson et al. 2017), the mass fractions are nearly unchanged and uncertainties in these values are not the dominant ones in our calculation. We calculate the virial temperature from the halo virial velocity, $v_{\text {vir, }}$ using

$$
T_{\mathrm{vir}}=\frac{\mu m_{p}}{2 k_{\mathrm{B}}} v_{\mathrm{vir}}^{2} \simeq 3.6 \times 10^{5} \mathrm{~K}\left(\frac{v_{\mathrm{vir}}}{100 \mathrm{~km} \mathrm{~s}^{-1}}\right)^{2} .
$$

Finally, we calculate the $\mathrm{H} \alpha$ recombination rate in units of $\mathrm{cm}^{3} \mathrm{~s}^{-1}$ for gas with temperature $T$ in units of $10^{4} \mathrm{~K}$ and density $\rho_{g}$ using the fitting function for the recombination rate,

$$
\alpha=10^{-13} \frac{2.274 T^{-0.659}}{1+1.939 T^{0.574}},
$$

from Pequignot et al. (1991).

To create mock observations, we select a sample of primary galaxies. We first randomly select lines of sight through the simulation volume that are presumed to be aimed at a background source that SDSS would have targeted. Cataloged halos within a projected separation of $1 \mathrm{Mpc}$ from this line of sight are considered hosts of potential primary galaxies, even if they are satellites within a larger halo. If the hosted galaxies meet the luminosity and size requirements $\left(10^{10}<L / L_{\odot}<\right.$ $10^{11}$ and $\left.2<R_{50} / \mathrm{kpc}<10\right)$ and if their apparent magnitude are above the SDSS targeting limit then they are accepted as primary galaxies. In detail, we use the observed galaxy luminosity-redshift relation of our primary galaxies to define the limiting luminosity at each redshift and then draw from the model to match that distribution. Because the mock catalog uses the luminosity-size distribution of the SDSS to set sizes, the joint luminosity-size distribution in the mock catalogs matches the SDSS by construction. Subsequently, any cataloged halo whose projected separation from the primary galaxies, $r_{p}$, is less than its virial radius, $r_{\mathrm{vir}}$, and whose recessional velocity is within $275 \mathrm{~km} \mathrm{~s}^{-1}$ of the primary galaxy's recessional velocity is an associated halo that could possibly be contributing $\mathrm{H} \alpha$ flux to the line-of-sight spectrum. For computational efficiency we neglect contributions from halos with $r_{p}>r_{\mathrm{vir}}$. We run some realizations where we change this criteria to $r_{p}>5 r_{\text {vir }}$ and find no detectable difference in the results. The SDSS magnitude limit directly affects the primary 


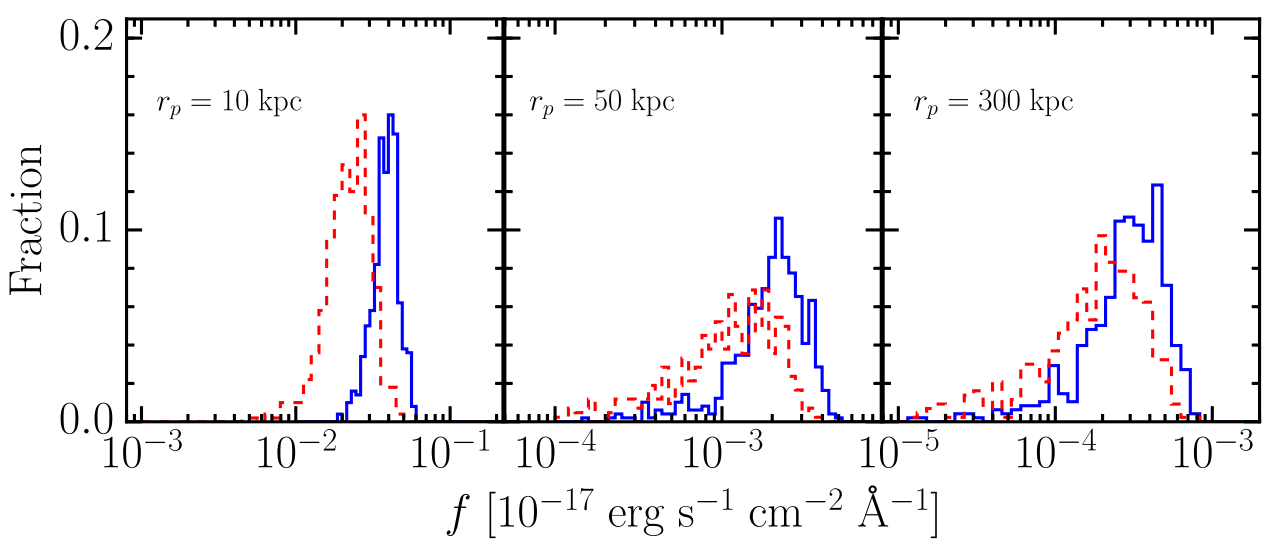

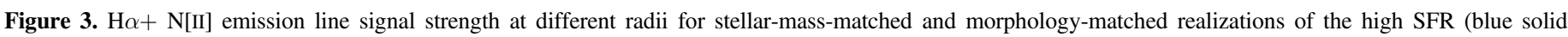
histogram) and low SFR galaxy samples (red dashed histogram).

halo selection function, but not the associated halo selection function, other than through correlations that may exist between the properties of associated halos and the primary ones.

We present the resulting model halo mass distributions for both the primaries and the associated halos in Figure 4. The halo mass of the primary galaxy is constrained within the range $10^{11}-10^{13} M_{\odot}$, reflecting the luminosity and half-light radius criteria we apply. The associated halo mass distribution is more representative of the universal halo distribution, with a preponderance of low-mass halos. The mean stellar mass, $M_{*}$, for the primary halos is $10^{10.88} M_{\odot}$ and is $10^{10.18} M_{\odot}$ for the associated halos.

Once we have our sample of primary and associated halos, we then integrate the square of the gas density, $\rho_{g}^{2}(r)$, projected along the line of sight, combine that measurement with the recombination rate, and derive a predicted $\mathrm{H} \alpha$ flux measurement. We truncate the density profile of each associated halo at its virial radius for computational efficiency. We find no significant difference in our results in a test run where we truncate the profiles at $5 r_{\text {vir. }}$. We calculate the flux for a $2^{\prime \prime}$ fiber (the BOSS fiber size) on the sky.

One significant difference between the data and the simulated measurements is that the observations include the contribution of $\mathrm{N}[\mathrm{II}]$. To account for this difference, we measure the $\mathrm{N}[\mathrm{II}]$ contribution from the stacked spectra for $r_{p}<50 \mathrm{kpc}$, where the lines are of sufficiently high $\mathrm{S} / \mathrm{N}$ that they are well-resolved, and measure that the $\mathrm{H} \alpha$ flux is 0.68 of the combined flux. Therefore, for our data and model comparisons, we multiply the modeled fluxes by 1.46 to account for $\mathrm{N}[\mathrm{II}]$ emission. The $\mathrm{H} \alpha / \mathrm{N}[\mathrm{II}]$ ratio can change as a function of radius (Miller \& Veilleux 2003), but this is beyond the scope of our models and the introduced uncertainty is likely to be lower than that introduced by other simplifying assumptions (see Section 3.3).

\subsection{Single Temperature Component}

The recombination rate is temperature-sensitive, so assumptions regarding the physical state of the gas drive the resulting emission measurements. We begin our first model by stipulating that the gas component is at a single temperature in each halo and that the temperature is the virial temperature. Because the typical virial velocity of a primary galaxy in our sample is $\sim 100 \mathrm{~km} \mathrm{~s}^{-1}$ or larger, the virial temperature is $\sim 10^{5}$ to $10^{6} \mathrm{~K}$. Hydrogen recombination at such high temperatures is

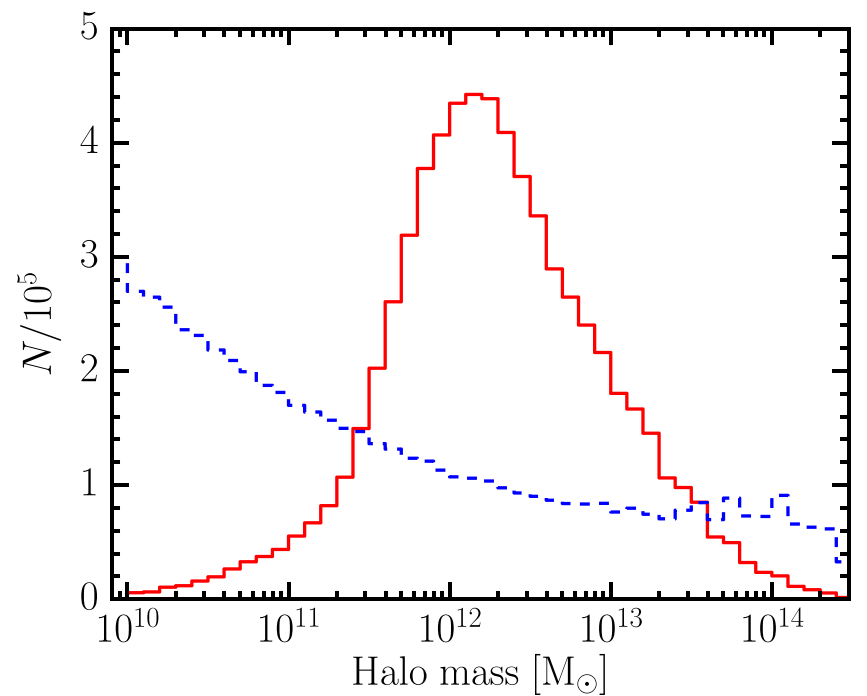

Figure 4. Halo mass distribution of the primary (solid red line) and secondary (dashed blue line) halos. The halo mass of the target galaxy is mostly limited to $10^{11}<M / M_{\odot}<10^{13}$ because of the luminosity and half-light radius selection criteria. The secondary halos are more representative of the full cosmological halo sample.

highly suppressed, resulting in simulated emission fluxes that are orders of magnitude lower than those observed (Figure 5). We conclude that either the observed emission has a completely different origin or our first model does not represent reality. This result is not surprising given the existing evidence for a multi-phase CGM (Tumlinson et al. 2017) and it strongly motivates a modification to our simple model. Because the shape of the theoretical radial emission profile is qualitatively similar to that observed, we are encouraged and suggest that only a simple modification is needed, presumably related to the assumption of a single high-temperature component.

\subsection{A Two Temperature Component Model}

Various absorption line studies (Werk et al. 2014; Tumlinson et al. 2017), theoretical models (Kereš et al. 2005; Ford et al. 2014), and our own work in Paper I conclude that much of the halo gas is at significantly lower temperatures than the virial temperature. In Paper I, we inferred that the emitting gas temperature is $\sim 12,000 \mathrm{~K}$. Therefore, we now explore a model where the gas is in two phases: one at the virial temperature and another at $12,000 \mathrm{~K}$. While we have a mechanism for 


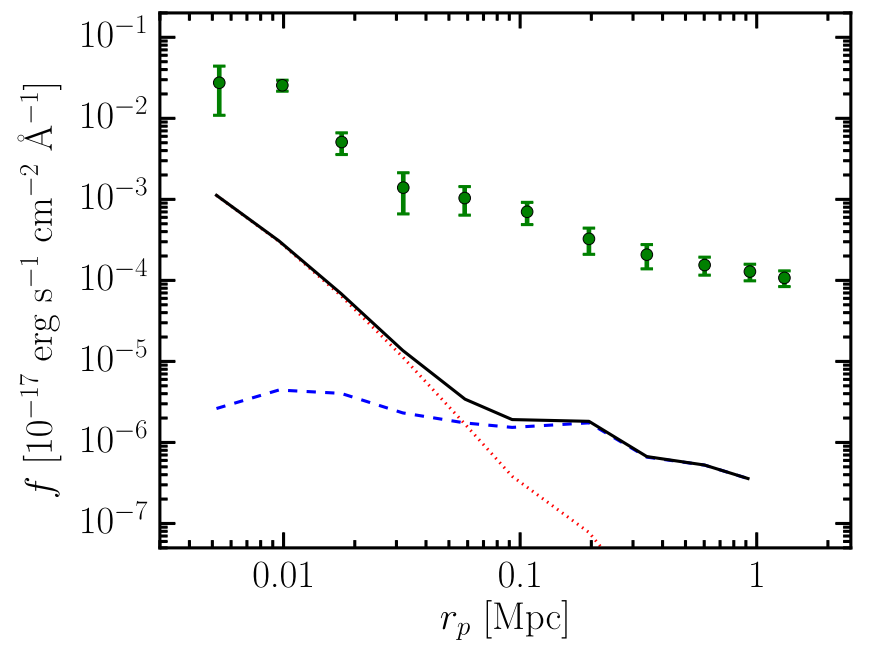

Figure 5. Comparison of data to a simulation with all halo gas at the corresponding halo virial temperature. The dotted red line represents the $\mathrm{H} \alpha$ $+\mathrm{N}[\mathrm{II}]$ emission arising in the primary halo of the target galaxy and the dashed blue line represents the emission from the associated halos. The solid black line represents the combined emission in the models. The green circles with error bars represent our measurements (Table 2). The predicted emission from this model is orders of magnitude lower than the data and even below the background level, $f_{B}$, at most radii.

computing the radial density profile of gas at the virial temperature within a dark matter halo, we do not have a simple way to estimate the corresponding distribution of $10^{4} \mathrm{~K}$ gas embedded within the hotter gas. We will assume that a fraction $C_{f}$ of the gas is in the cold component and that it follows the same radial profile as the hotter gas. The last potential complication in our simple model is whether this gas is clumped. If it is clumped, then the higher local density knots will emit more recombination radiation than if that same quantity of gas is distributed smoothly. For now we will assume a smooth distribution, but we will return to this issue later.

In Figure 6 we show our first attempt at fitting such models. We consider only the contribution from the primary galaxy halo itself, which is indicated by the red dotted line for an adopted $C_{f}=0.27$, and the contribution from the background, $f_{B}$. We determine $C_{f}$ by minimizing $\chi^{2}$. The model fits the data well out to $\sim 50 \mathrm{kpc}$ and at $r_{p}>1 \mathrm{Mpc}$. Within $50 \mathrm{kpc}$, the one outlier is the second innermost point, which at $r_{p} \sim 10 \mathrm{kpc}$ is perhaps unlikely to be well described with this simple model. Only considering the data out to $\sim 50 \mathrm{kpc}, \chi^{2}=7.02$, for which the model cannot be ruled out with greater than $80 \%$ confidence. However, the $\chi^{2}$ value for $r_{p}<1 \mathrm{Mpc}$ is 17.87 , for which the model can be ruled out with $97 \%$ confidence.

The model principally fails to reproduce the fluxes observed at intermediate radii. These fluxes are significantly larger than the background uncertainty and cannot be reproduced by modeled emission from the central halo. Increasing $C_{f}$ would raise the entire red curve, thereby grossly overproducing the flux at $r_{p}<50 \mathrm{kpc}$. Models with a single halo component plus a systematic background floor cannot reproduce the observations.

In Figure 7 we present the next iteration of the models, now including the contribution from correlated, nearby halos. Because the primary and associated halos have a different mass distribution, we expect the characteristic $C_{f}$ 's to differ. Simulations of the halo gas show such a variation with the galaxy's stellar mass (Tumlinson et al. 2017). The average

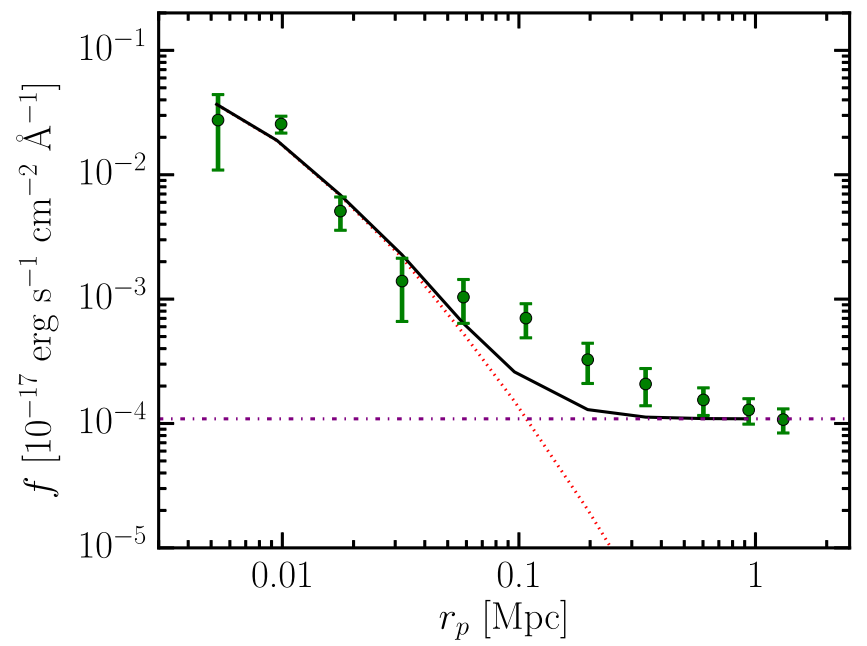

Figure 6. Comparison of data and a simulation with fraction, $C_{f}$, of the halo gas at $12,000 \mathrm{~K}$ set to 0.27 and the remainder at the corresponding halo virial temperature for all halos. In this figure we show only the $\mathrm{H} \alpha+\mathrm{N}[\mathrm{II}]$ emission arising from the primary halo (dotted red line) and the systematic limit on our measurement of the background, $f_{B}$ (horizontal line). Together (solid black line), these two components match the observed emission profile (green circles with error bars) at small and large $r_{p}$, but fail at intermediate $r_{p}$, illustrating the need for an additional component.

stellar mass for our modeled primary galaxies is $10^{10.88} M_{\odot}$ and is $10^{10.18} M_{\odot}$ for our secondary galaxies. Therefore, in our fitting we allow for different $C_{f}$ values for the primary and secondary halos. Our best-fit values are $C_{f}=0.26$ and 0.34 for the primary and secondary halos, respectively, and the fit (left panel of Figure 7) is excellent. Alternatively, we can adopt $C_{f}$ from independent hydrodynamical simulations of galaxy halos (Ford et al. 2014), which provide a relationship between $C_{f}$ and stellar mass. That relation predicts that the appropriate $C_{f}^{\prime}$ 's are 0.19 and 0.38 , respectively. Those values also produce a good fit, and have the added virtue of removing all fitting freedom.

With or without fitting $C_{f}^{\prime}$ s, we now obtain a good match to the observations and conclude that the emission profile inflection at $r_{p} \sim 50 \mathrm{kpc}$ can be explained as the onset of dominance by associated halos. The best-fit matches the observations quite well, with a $\chi^{2}$ of 9.17 for $r_{p}<1 \mathrm{Mpc}$. The hypothesis that the decrease in $\chi^{2}$, from 17.87 to 9.17 , resulting from the addition of the added freedom of allowing a second component, is consistent with random chance can be rejected with $99.8 \%$ confidence. We conclude with high confidence that there is evidence for the contribution from associated halos.

At this point, a natural question arises regarding the nature of these associated halos. Are they principally satellites of the primary, parents of the primary, or distinct second halos? For various reasons we consider satellites to be an unlikely significant source of $\mathrm{H} \alpha$ flux. First, the SDSS target itself could not be a satellite because of our redshift difference requirement, which corresponds to a line-of-sight difference of $\sim 200 \mathrm{Mpc}$. Second, the odds that satellites are interlopers along the lines of sight are low because of their small cross sections. Third, we have eliminated lines of sight with significantly strong $f$ and therefore a few strongly emitting satellites could not be the source of the signal. Finally, most satellites within $100 \mathrm{kpc}$ of $L_{*}$ galaxies are gas-poor. The models confirm this conclusion, with virtually no flux originating in halos of $M<2.5 \times 10^{11} M_{\odot}$, a limit that is 10 times the resolution limit of the UNIVERSEMACHINE mock 


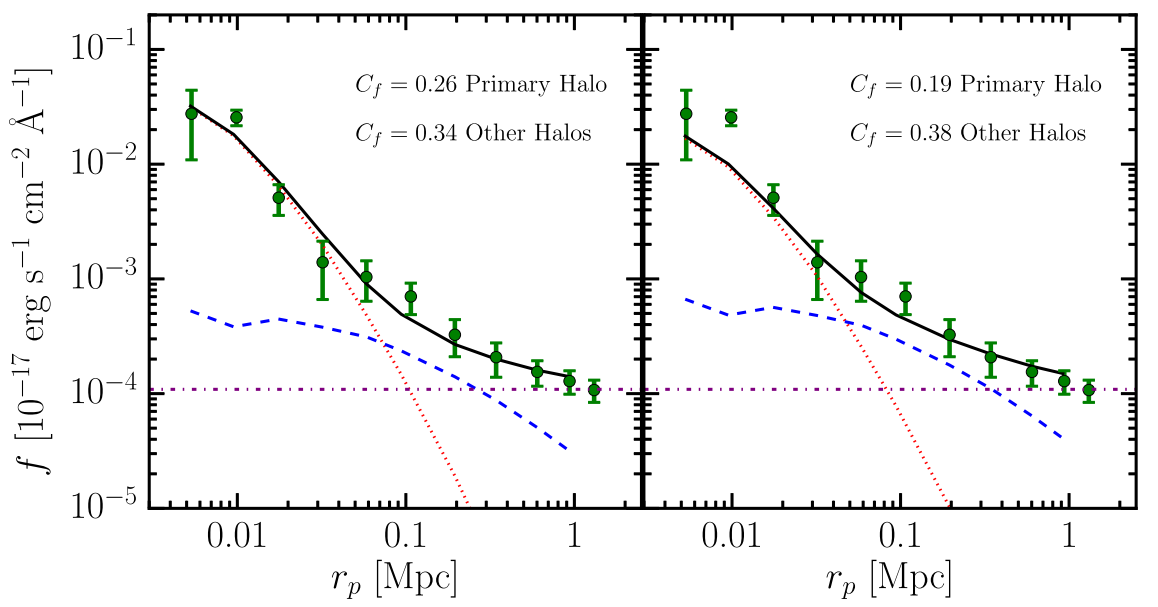

Figure 7. Comparison of data to simulations. Models assign different $C_{f}$ 's to primary and associated halos, but all have the smoothly distributed cold halo gas temperature set at $12,000 \mathrm{~K}$ and the remainder of the gas at the corresponding halo virial temperature for all halos. In this figure we show the $\mathrm{H} \alpha+\mathrm{N}[\mathrm{II}]$ emission arising from the primary halo (dotted red line) and the associated halos (blue dashed line) and the systematic limit on our measurement of the background, $f_{B}$ (horizontal line). Together (solid black line), these three components are compared to the observed emission profile (green circles with error bars). The left panel shows model results from our minimization of $\chi^{2}$ while varying $C_{f}$ 's. The right panel shows the model results when we adopt the $C_{f}$ from the relation between $C_{f}$ and stellar mass produced by independent hydrodynamical simulation (Ford et al. 2014).

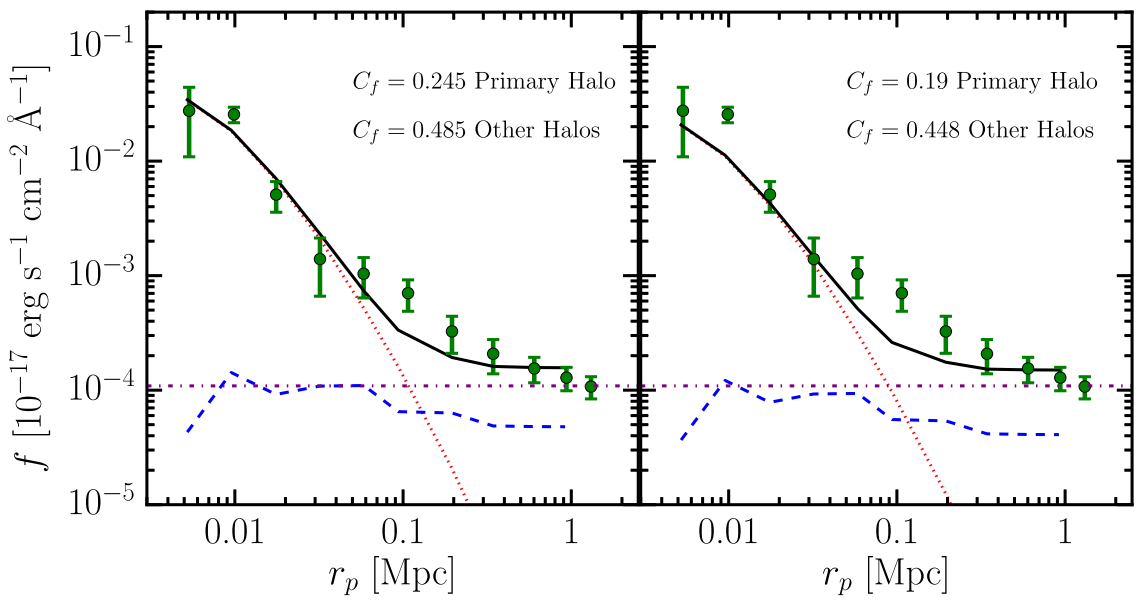

Figure 8. Comparison of data to simulations that exclude primary galaxies that are satellites within larger halos. We refit $C_{f}$ 's for primary and secondary halos. In this figure we show the $\mathrm{H} \alpha+\mathrm{N}[\mathrm{II}]$ emission arising from the primary halo (dotted red line) and the secondary halos (blue dashed line) and the systematic limit on our measurement of the background, $f_{B}$ (horizontal line). Together (solid black line), these three components are compared to the observed emission profile (green circles with error bars). The left panel shows model results from our minimization of $\chi^{2}$ while varying $C_{f}$ 's. Right panel shows the model results when we adopt the $C_{f}$ from the relation between $C_{f}$ and stellar mass produced by independent hydrodynamical simulation (Ford et al. 2014).

catalogs. Considering the second option, we find that about $20 \%$ of the primary galaxies in the simulations are satellite galaxies within group and cluster size halos. In our current model, these parent halos contribute significantly to the excess flux above the single halo expectation at intermediate radii (50-100 kpc), as we show in Figure 8. Refitting $C_{f}$ for the case with no satellites results in values of $C_{f}>0.4$ for the associated halos, but does not reproduce the flux at these intermediate radii. Lastly, considering the third option, we find that distinct second halos contribute a modest amount, although that amount might be critical to explaining the flux detected at $r_{p}>200 \mathrm{kpc}$. Obviously, we are approaching the limits of the current measurements, but the models do suggest that the second halo term could be detected. Definitively measuring the second halo contribution will require improved precision.

So far, our simple model seems sufficient to explain the observations. However, there are clear complications that must be present. First, we have adopted a single temperature for the cold component, not just from halo to halo but also radially within each halo. The recombination rate for the adopted temperature of $12,000 \mathrm{~K}$ is $6.40 \times 10^{-14} \mathrm{~cm}^{3} \mathrm{~s}^{-1}$ but changes nearly inversely with temperature, ranging from $1.30 \times 10^{-13} \mathrm{~cm}^{3} \mathrm{~s}^{-1}$ at $6000 \mathrm{~K}$, to $3.04 \times 10^{-14} \mathrm{~cm}^{3} \mathrm{~s}^{-1}$ at $24000 \mathrm{~K}$. This dependence means that relatively modest, factor of 2, discrepancies in emission flux could be explained by plausible temperature variations within what we refer to as the cold component.

Second, we have adopted a model with a smooth density distribution of cold gas. The cold gas is likely to be clumped and the emission flux depends on density squared. Stacking a large number of systems is presumably helping to average over the most discrepant cases, but a systematic bias in the modeled fluxes, depending on the fraction of gas that is in clumps, could be present.

To allow for more generality in our discussion, we define an emission factor, $\epsilon=C_{f}(T / 12,000 \mathrm{~K})^{-0.5}(c / 1)$, where $c$ is a gas clumping factor. Our constraints above are in practice on the emission factor $\epsilon$, and the cold fraction $C_{f}$ could vary as 
long as it is balanced by corresponding changes in the gas temperature $T$ and/or the clumping factor $c$ from our assumptions above.

Third, our model makes no attempt to distinguish between primary galaxies that are the dominant galaxy in their halo versus those that are satellites within a more massive halo. It is entirely reasonable to expect the satellite galaxies to have different gaseous halo properties than the isolated ones. To first order, if we presume that the satellites have lost their gaseous reservoir, that would simply depress the predicted $f$ by the fraction of satellites in the sample, $\sim 20 \%$. Such a difference could easily be compensated in the models by a commensurate increase in the clumping discussed previously. As such, even though this discussion clearly points to a shortcoming of our models, the effect is likely to be well within our rather large uncertainties.

The effect of nearby halos on measurements of halo gas has been explored before in theoretical work. For example, Nelson et al. (2018) explored the contributions from other halos to the column density of absorption line systems arising from highly ionized oxygen O VI, O VII, and O VIII gas. They found in their simulations that the contribution from second halos dominate in the 3D profiles $50-100 \mathrm{kpc}$ beyond $r_{\text {vir }}$ for halos of $10^{12} M_{\odot}$. A comparison between emission and absorption line properties of different tracers holds promise as a powerful model discriminant because of the different dependencies of the two measurements on the temperature and density of the gas.

The need for a more complex model can be seen clearly when we examine our model further. In the case with no fitting, we adopted the $C_{f}$ from Ford et al. (2014) corresponding to the mean stellar mass of the associated halos (log $\left.\left(M_{*} / M_{\odot}\right)=10.18\right)$. However, in that model the halo emission, flux-weighted mean stellar mass, which might be the appropriate one to use because the bulk of the flux is coming from such systems, is significantly higher $\left(\log \left(M_{*} / M_{\odot}\right)=10.72\right)$. For this high a stellar mass the Ford et al. (2014) study predicts a much lower $C_{f}$. This choice of $C_{f}$ leads to a much lower flux from the associated halos, which results in an emission profile that does not match that observed. Given this conundrum, we also ran a model where the $C_{f}$ of each individual associated halo was adjusted using the Ford et al. (2014) relation. This model also failed to reproduce the radial profile at intermediate radii.

There are two ways to resolve this discrepancy. First, the Ford et al. (2014) relation between $M_{*}$ and $C_{f}$ may be incorrect. This is an interesting possibility in that our observations may be telling us how the hydrodynamical models need to be improved. Second, and perhaps even more likely, discrepancies may be due to variations in $c$ and $T$ as a function of $M_{*}$ (as well as radius and environment) that are not accounted for in our simple model. Again, this may lead to greater insight into the CGM, particularly if some of the degeneracies involved in calculating the emission fluxes can be broken by including absorption line constraints.

\subsection{Higher-order Tests of the Model}

So far, we have demonstrated that the simple fitted model matches the data from the full sample and reveals that the galaxy's neighboring halos are responsible for the dominant contribution to $\mathrm{H} \alpha+\mathrm{N}[\mathrm{II}]$ emission at intermediate $r_{p}$. We now investigate what the model predicts about how the primary galaxy's properties correlate to the emission profile of the gaseous halo and how those predictions compare to the observations.

For the comparison of the halo properties as a function of the SFR of the primary galaxy, we divide the entire model sample into a high SFR subsample (SFR $\left.>0.7 M_{\odot} \mathrm{yr}^{-1}\right)$ and low SFR subsample (SFR $\leqslant 0.7 M_{\odot} \mathrm{yr}^{-1}$ ) and follow the same massmatching procedure as we did for the observational sample. Rather than showing the resulting $f$ distribution, as we do for the data, for the models we only show the resulting mean values in Figure 2.

There are some successes in the model predictions, for example the agreement between the predicted and observed $f$ values for the high and low SFR galaxies at large $r_{p}$. However, the failures may be more illuminating. The models fail for both the high and low SFR galaxies at small $r_{p}$, by underpredicting and overpredicting the emission flux respectively. In essence, the model predicts that the halo emission from high and low SFR galaxies is much more similar at these radii than we observe it to be. Clearly, there are important physical details, the role of feedback and the nature of escaping ionizing radiation, that are not included in our simple models. One can imagine that feedback has removed at least some of the gas reservoir in early type galaxies, leading to lower emission fluxes than we predict, and that the inner gaseous halos are slightly more massive or denser around star-forming galaxies, leading to higher emission fluxes than we predict. Perhaps most disappointing is that the models fail to qualitatively reproduce the reversal observed between the higher fluxes at small $r_{p}$ for high SFR galaxies and higher fluxes at large $r_{p}$ in low SFR galaxies. We had expected this behavior to be the result of correlations between SFR and environment, but the model does not confirm this hypothesis.

These discrepancies warrant further investigation with more realistic models and could reflect problems in our simplified halo gas characterizations or in how galaxies with certain SFR or morphology are assigned to halos. In either case, further study should be illuminating.

\section{Conclusions}

Using over 8 million spectra that intersect the halos of nearly half a million nearby galaxies, we find that the radial profile of $\mathrm{H} \alpha+\mathrm{N}[\mathrm{II}]$ emission line around normal galaxies extends well beyond $100 \mathrm{kpc}$ in projected radius and has an inflection at a projected radius of $\sim 50 \mathrm{kpc}$.

We also find trends in the halo emission line profiles with properties of the primary galaxy. At small projected radius, $r_{p} \sim 10 \mathrm{kpc}$, the line emission flux in the halos of high SFR or disk-dominated galaxies is a few times higher than that in the low SFR or spheroid-dominated galaxies. At $r_{p} \sim 50 \mathrm{kpc}$ the emission flux profiles become somewhat more similar and at even larger $r_{p}, \sim 300 \mathrm{kpc}$, the behavior reverses, with the high SFR and disk-dominated galaxies having lower levels of emission. These results are for stellar-mass-matched samples. When we control for both stellar mass and morphology, we still find differences in the halo emission that are related to the SFR of the central galaxy. These results highlight the connectivity between the central galaxy and the surrounding CGM, extending beyond its immediate vicinity. The emission profile, and its dependence on galaxy properties, promises to be a key constraint on models of the CGM/galaxy connection.

To further understand the emission profile inflection and relationships between the primary galaxy and the emission 
profile, we constructed a simple, but cosmologically motivated, halo model, where each halo is populated with gas using a hydro-equilibrium isothermal NFW profile. The gas is split into a hot component at the virial temperature and a cooler component, $T=12,000 \mathrm{~K}$. This model matches the emission profile from the full sample extremely well, whether we fit the fraction of cold gas in primary and secondary halos (0.26 and 0.34 ) or we adopt values published previously derived from hydrodynamical simulations $(0.19$ and 0.38$)$. We demonstrated statistically that the contributions from associated halos are necessary to produce the excellent match. At $r_{p}>50 \mathrm{kpc}$, the emission flux is primarily coming from these neighboring halos. This result explains both the inflection in the radial emission line profile and the decreasing correlation we find between the emitted flux and the properties of the primary galaxy for increasing $r_{p}$.

Our model is manifestly oversimplified because we ignore temperature variations, assume a smoothly varying cold gas density profile, and procedurally assign galaxies to dark matter halos. As such, perhaps it is not too surprising that despite the successes described above, the model fails to reproduce more detailed observational findings that we discuss. This failure likely indicates that the cold gas properties (fraction, temperature, density) depend on aspects of galaxy formation and evolution that have not been included in our simple model. Either way, additional, more realistic modeling of the halo emission line fluxes has the potential to help us understand important aspects of galaxy structure and evolution. We provide our measurements as a constraint for future theoretical models.

D.Z. and H.Z. acknowledge financial support from NASA ADAP NNX12AE27G and NSF grant AST-1311326. The authors gratefully acknowledge Yinzhe Ma and Houjun Mo for helpful discussions, and the SDSS-III team for providing a valuable resource to the community.

Funding for SDSS-III has been provided by the Alfred P. Sloan Foundation, the Participating Institutions, the National Science Foundation, and the U.S. Department of Energy Office of Science. The SDSS-III web site is http://www.sdss3.org/.

SDSS-III is managed by the Astrophysical Research Consortium for the Participating Institutions of the SDSS-III Collaboration including the University of Arizona, the Brazilian Participation Group, Brookhaven National Laboratory, Carnegie Mellon University, University of Florida, the French Participation Group, the German Participation Group, Harvard University, the Instituto de Astrofisica de Canarias, the Michigan State/Notre Dame/JINA Participation Group, Johns Hopkins University, Lawrence Berkeley National Laboratory, Max Planck Institute for Astrophysics, Max Planck Institute for Extraterrestrial Physics, New Mexico State University, New York University, The Ohio State University, Pennsylvania
State University, University of Portsmouth, Princeton University, the Spanish Participation Group, University of Tokyo, University of Utah, Vanderbilt University, University of Virginia, University of Washington, and Yale University.

\section{ORCID iDs}

Huanian Zhang (iD https://orcid.org/0000-0002-0123-9246 Dennis Zaritsky (iD https://orcid.org/0000-0002-5177-727X Peter Behroozi (iD https://orcid.org/0000-0002-2517-6446

\section{References}

Adams, J. J., Uson, J. M., Hill, G. J., \& MacQueen, P. J. 2011, ApJ, 728, 107 Alam, S., Albareti, F. D., Allende Prieto, C., et al. 2015, ApJS, 219, 12 Behroozi, P. S., Conroy, C., \& Wechsler, R. H. 2010, ApJ, 717, 379 Behroozi, P. S., Wechsler, R. H., \& Wu, H.-Y. 2013, ApJ, 762, 109 Behroozi, P. S., Wechsler, R. H., Wu, H.-Y., et al. 2013, ApJ, 763, 18 Bellhouse, C., Jaffé, Y. L., Hau, G. K. T., et al. 2017, ApJ, 844, 49 Bland-Hawthorn, J., Freeman, K. C., \& Quinn, P. J. 1997, ApJ, 490, 143 Bregman, J. N. 2007, ARA\&A, 45, 221

Bridge, J. S., Hayes, M., Melinder, J., Östlin, G., \& Gronwall, C. 2017, AAS Meeting, 229, 222.01

Brinchmann, J., Charlot, S., White, S. D. M., et al. 2004, MNRAS, 351, 1151

Capelo, P. R., Natarajan, P., \& Coppi, P. S. 2010, MNRAS, 407, 1148

Christlein, D., Zaritsky, D., \& Bland-Hawthorn, J. 2010, MNRAS, 405, 2549

Dicaire, I., Carignan, C., Amram, P., et al. 2008, AJ, 135, 2038

Ford, A. B., Davé, R., Oppenheimer, B. D., et al. 2014, MNRAS, 444, 1260

Fumagalli, M., Haardt, F., Theuns, T., et al. 2017, MNRAS, 467, 4802

Gallazzi, A., Charlot, S., Brinchmann, J., White, S. D. M., \& Tremonti, C. A. 2005, MNRAS, 362, 41

Hayes, M., Östlin, G., Duval, F., et al. 2014, ApJ, 782, 6

Hayes, M., Östlin, G., Schaerer, D., et al. 2013, ApJL, 765, L27

Hlavacek-Larrondo, J., Marcelin, M., Epinat, B., et al. 2011, MNRAS, 416, 509

Kauffmann, G., Heckman, T. M., White, S. D. M., et al. 2003a, MNRAS, 341,33

Kauffmann, G., Heckman, T. M., White, S. D. M., et al. 2003b, MNRAS, 341,54

Kereš, D., Katz, N., Weinberg, D. H., \& Davé, R. 2005, MNRAS, 363, 2

Klypin, A., Yepes, G., Gottlöber, S., Prada, F., \& Heß, S. 2016, MNRAS, 457, 4340

McGaugh, S. S., Schombert, J. M., de Blok, W. J. G., \& Zagursky, M. J. 2010, ApJL, 708, L14

Miller, S. T., \& Veilleux, S. 2003, ApJS, 148, 383

Navarro, J. F., Frenk, C. S., \& White, S. D. M. 1997, ApJ, 490, 493

Nelson, D., Kauffmann, G., Pillepich, A., et al. 2018, MNRAS, 477, 450

Pequignot, D., Petitjean, P., \& Boisson, C. 1991, A\&A, 251, 680

Planck Collaboration, Ade, P. A. R., Aghanim, N., et al. 2013, A\&A, 557, A52

Rodríguez-Puebla, A., Behroozi, P., Primack, J., et al. 2016, MNRAS, 462,893

Sérsic, J. L. 1968, Atlas de Galaxias Australes (Cordoba: Observatorio Astronomico)

Simard, L., Mendel, J. T., Patton, D. R., Ellison, S. L., \& McConnachie, A. W. 2011, ApJS, 196, 11

Spitzer, Jr. L. 1956, ApJ, 124, 20

Tumlinson, J., Peeples, M. S., \& Werk, J. K. 2017, ARA\&A, 55, 389

Werk, J. K., et al. 2014, ApJ, 792, 8

Zaritsky, D., \& Courtois, H. 2017, MNRAS, 465, 3724

Zhang, H., Zaritsky, D., Zhu, G., Ménard, B., \& Hogg, D. W. 2016, ApJ, 833,276 